\title{
Studies in the History of Probability and Statistics. XXXV Multiple decrements or competing risks
}

\author{
BY HILARY L. SEAL \\ Départment de Mathématiques, Ecole Polytechnique Fédérale de Lausanne, \\ Switzerland
}

\section{SUMMARY}

Given two states $A$ and $B$ such that individuals in state $A$ have mutually exclusive probabilities, possibly dependent on the time spent in state $A$, of leaving that state because of (i) death, or (ii) passage to state $B$, what is the probability of an individual passing to state $B$ and dying there within a given period? This problem has been of great interest and importance to actuaries for over 100 years and the solutions of their professional contemporaries have appeared in their textbooks. Twenty-five years ago statisticians felicitously named the technique the theory of competing risks (Neyman, 1950) and developed the formulae ab initio in the framework of Markov processes. Consideration of the problem started with Daniel Bernoulli's smallpox mathematics and continued with pension fund financing involving the payment of annuities to invalid lives. Most of the currently accepted techniques were developed during the nineteenth century. This article reviews the history both of actuarial and statistical contributions to the literature.

Some key words: Actuarial mathematics; Competing risk; Multiple decrement.

\section{INocolation agaINST SMaLIPOX}

In order to conform with the abundant actuarial literature of this century it is desirable to utilize a notation due to the Russian actuary Hamza (1900). Write $l_{x}$ for the expected number of survivors at age $x$ from $l_{0}$, an initial group of entrants into state $A$ at age zero which need not be the 'real' zero of birth. At age $x$ this expected number has become subdivided into $l_{x}^{\text {aa }}$ expected in state $A$ and $l_{x}^{k i}$ in state $B$, that is

$$
l_{x}=l_{x}^{a a}+l_{x}^{i i}, \quad d l_{x}=d l_{x}^{a a}+d l_{x}^{i i} .
$$

It is, or course, preferable to write $l_{0}=1$ and make $l_{x}$ the probability of survival to at least age $x$. This is not, however, the way in which the earlier writers viewed $l_{x}$. While random variations were envisaged in the actual number of survivors to age $x$ it was assumed that this number was so large that it converged to the 'true' value. Nowadays we can avoid ambiguity by referring to $l_{x}$ as the expected number of survivors at age $x$. Now write $\mu_{x}^{a}$ for the force of decrement, or conditional instantaneous probability of exit, from state $A$, consisting of $\mu_{x}^{a a}$, the force of mortality, and $v_{x}$ the force of passage to $B$. Let $\mu_{x}^{i}$ be the force of mortality affecting individuals in state $B$. No other type of movement from state $B$ is envisaged. Then, considering the changes in the groups $A$ and $B$ in the infinitesimal interval of time following age $x$,

$$
-d l_{x}^{a a}=l_{x}^{a a} \mu_{x}^{a} d x=l_{x}^{a a}\left(\mu_{x}^{a a}+\nu_{x}\right) d x, \quad d l_{x}^{i t}=l_{x}^{a a} \nu_{x} d x-l_{x}^{i d} \mu_{x}^{i} d x
$$

Hence, from (1),

$$
-d l_{x}=l_{x}^{a a} \mu_{x}^{a a} d x+l_{x}^{i d} \mu_{x}^{i} d x
$$


expressing the fact that deaths in the whole community are composed of deaths from $A$ plus deaths from $B$. This can be rewritten as

$$
-d l_{x}=l_{x} \mu_{x}^{a a} d x+l_{x}^{i d}\left(\mu_{x}^{i}-\mu_{x}^{a a}\right) d x=l_{x} \mu_{x}^{a a} d x+l_{x}^{i d} \phi_{x} d x,
$$

and, on substituting this implicit value of $\mu_{x}^{a a}$ in the first equation (2), we have

$$
d d_{x}^{a a}=\frac{l_{x}^{a a}}{l_{x}}\left(d l_{x}+l_{x}^{i i} \phi_{x} d x\right)-l_{x}^{a a} \nu_{x} d x
$$

The solution, derived by Richard (1846, p. 79) without reference to any originator, is

$$
l_{x}^{a a}=l_{x} \exp \left\{-\int_{0}^{x}\left(\mu_{u}^{a}-\mu_{u}^{i}\right) d u\right\}\left[1+\int_{0}^{x}\left(\mu_{u}^{i}-\mu_{u}^{a a}\right) \exp \left\{-\int_{0}^{u}\left(\mu_{w}^{a}-\mu_{w}^{i}\right) d w\right\} d u\right]^{-1} .
$$

The differential equation (5) is essentially that obtained by Daniel Bernoulli (1766) in his pathbreaking application of infinitesimal methods to a problem in probability (Westergarard, 1932, chapter IX). It is included in Laplace's textbook (1812, Livro II, Art. 36). Bernoulli's state $A$ consisted of individuals who had never had smallpox while state $B$ comprised those who had contracted smallpox and would either die from it very soon, or survive and no longer be subject to that disease. In Bernoulli's, and Laplace's, model there is thus an immediate heavy mortality aflecting those who catch smallpox followed by normal mortality, $\mu_{x}^{a a}$, thereafter. Both Bernoulli and Laplace took account of this slight modification in our general model by replacing the second term, $l_{x}^{i i} \phi_{x}$, of (4) by $l_{x}^{a a} v_{x} \rho_{x}$, the deaths from smallpox, $\rho_{x}$ being the proportion of smallpox sufferers at age $x$ who die from that disease. In Bernoulli's time there were no age-specific statistics to estimate $\nu_{x}$ and $\rho_{x}$ and the best he could do was to guess them both equal to $0 \cdot 125$, independent of age. Most of Bernoulli's 45-page memoir is concerned with the legitimacy of this assumption and the statistical conclusions to which it led. His only mathematical formulae consisted of the development of $(6)$ which in his special case, with $\nu_{x}-\phi_{x}=1 / n$ and $\phi_{x}=-1 /(n m)$, became

$$
l_{x}^{a a}=l_{x} e^{-x / n}\left\{1-\frac{1}{m}\left(1-e^{-x / n}\right)\right\}^{-1},
$$

and the corresponding expression for the proportion of the $l_{x}^{a a}$ individuals who have not had smallpox, namely

$$
l_{x}^{a a} / \exp \left(-\int_{0}^{x} \nu_{u} d u\right)
$$

Bernoulli pointed out that with $v_{u}=1 / n$ and the foregoing expression for $l_{x}^{a a},(8)$ approaches $m /(m-1)$ as $x \rightarrow \infty$. With his choice of $m=n=8$, he evaluated (7) and (8) for $x=1(1) 24$ "and $x=1(1) 25$, respectively, and used Halley's Breslan table for $l_{x}$, overlooking that Halley had calculated $\int l_{y}^{\prime} d y$ with limits $x$ and $x+1$, and not $l_{x}$ (Westergarard, 1932). Laplace, also considering inoculation for smallpox, in effect put $\nu_{x}-\phi_{x}=\zeta_{x}$ and $\phi_{x}=-\xi_{x}$ and reached:( $(\theta)$ without any attempt at numerical evaluation.

D'Alembert wrote at somejlength (1761) on the difficulty of comparing 'un danger présent', namely death from inoculation, then estimated at about $1 / 300$, with 'un danger éloigné', eventual death from smallpox, and argued that a comparison of life expectancies was not the way to proceed. Todhunter (1865, Arts. 479-88, 506-7 and 516-25) provides a review of this author's work and in order to derive his principal relation it is convenient to change our problem and notation slightly.

Write $\mu_{x}^{(i)}$ for the force of mortality at age $x$ on account of cause of death $i$ and $\mu_{x}^{(-i)}$ for the force of $m$ rtality at that age due to all causes of death other than $i$. With $l_{x}$ having the same 
meaning as before

and, in an obvious notation,

$$
\begin{gathered}
-d l_{x}=l_{x}\left(\mu_{x}^{(i)}+\mu_{x}^{(-i)}\right) d x \\
-d l_{x}^{(-i)}=l_{x}^{(-i)} \mu_{x}^{(-i)} d x,
\end{gathered}
$$

which leads to

On integrating, we obtrin

$$
d l_{x}^{(-i)} / l_{x}^{(-i)}=d l_{x} l_{x}+\mu_{x}^{(i)} d x .
$$

$$
l_{x}^{(-\mathfrak{l})}=l_{x} \exp \left(\int_{0}^{x} \mu_{u}^{(i)} d u\right)
$$

This, equivalent to (8) but free of $\nu_{x}$, is the relation obtained by d'Alembert (1761) for the expected number of individuals in $l_{x}$ who have not had smallpox, except that $\mu_{x}^{(i)} d x$ was there replaced by its equivalent, the differential $d u_{x} / l_{x}$, where $u_{x}$ is the aggregate expected number of deaths prior to age $x$ on account of cause $i$ since the $l_{0}$ children were born. Although d'Alembert proposed to cumulate the deaths from smallpox age by age Todhunter (1865) stated that (10) was 'not of practical use because the value of the integral ... is not known'. On the other hand Laplace (1812) had explained that approximate values of $\mu_{x}^{(i)}$ at successive integer ages conld be obtained by dividing the number of deaths from cause $i$ at age $x$ by the number of survivors from $l_{0}$ infants between ages $x-\frac{1}{2}$ and $x+\frac{1}{2}$. The integral in (10) could then be obtained by trapezoidal quadrature. Karn (1931), who provides an excellent account of the history of inoculation for smallpox, used a similar estimation procedure except that she approximated the differential coefficient $d u_{x} / d x$ at age $x$ by means of the operator relation $D=\log (1+\Delta)$ through the term in $\Delta^{3}$. She then used the Euler-Maclenrin expension for the integral through second differences.

We observe that if all the causes of death are denoted by $i_{j}(j=1, \ldots, k)$ relation $(10)$ can be written

$$
l_{x}=\prod_{j=1}^{k} \exp \left(-\int_{0}^{x} \mu_{u}^{(i j)} d u\right)=\exp \left(-\sum_{j=1}^{k} \int_{0}^{x} \mu_{\mathfrak{u}}^{(\mathcal{L})} d u\right)
$$

This formula first appeared in Cournot (1843, p. 317). The English actuary Makeham apparently discovered it independently (1867) and used it to interpret (1874) the foregoing developments of Bernoulli, d'Alembert and Laplace. Kanner (1871) derived it again, without using single letters for the forces of exit, also without referring to any precursors.

Two other athors contributed to the inoculation controversy by producing statistics and questioning the age independence of Bernoulli's $m$ and $n$. One was Lambert (1772, Chapter vII) and the other Duvillard (1806; Quiquet, 1934, Dossier XIII); neither added anything to the foregoing theory.

Finally, we mention a paper that foreshadowed the developments of the mid-nineteenth century. Trembley (1798) sought to derive Bernoulli's relation (7) without using infinitesimal calculus and to generalize it to apply to age-dependent $n^{-1}$ and $m^{-1}$ which we will here write as $w_{x}$ and $q_{x}^{i}$, respectively. Although Trembley developed his formulae by years of age and shifted all his smallpox cases to the year-end he emphesized, and demonstrated by numerical examples, that the unit of time could be much smaller, in particular 1/256th of a year. Trembley's result, obtained by simple arguments, wes

and could be rewritten in the form

$$
l_{x+1}^{a a}=\frac{l_{x}^{a a} l_{x} p_{x}\left(1-w_{x}\right)}{l_{x}-l_{x}^{a a} w_{x} q_{x}^{i}}
$$

$$
p_{x}^{a a}=\frac{p_{x}\left(1-w_{x}\right)}{1-l_{x}^{a a} w_{x} q_{x}^{i} / l_{x}}
$$

where $p_{x}^{a a}=l_{x+1}^{a a} / l_{x}^{a a}$. This relation would provide the series $l_{x}^{a a}$ for $x=1,2, \ldots$ in succession and 
Trembley pointed out that when $w_{x}=1 / n$ and $q_{x}^{i}=1 / m$ an inductive argument produces

and this tends to (7) when $n \rightarrow \infty$.

$$
l_{x}^{a a}=\frac{l_{x}\left(1-n^{-1}\right)^{x}}{1-m^{-1}+m^{-1}\left(1-n^{-1}\right)^{x}}
$$

Trembley concludes his memoir by citing Lambert's (1772) and another series of values of $w_{x}$ and $q_{x}^{i}$ (Todhunter, 1865, Art. 787). The differences between the series are considerable.

\section{INSURANOE AgATNST PERMaNENT INVALIDITY}

The conclusion to be drawn from the foregoing is that the basic mathematics of what we now call the continuous time version of a Markov chain model (Bartholomew, 1973) were developed during the eighteenth century. Moreover the methods were reported in the probability texts of the early nineteenth century. Although the concept of state $B$ as that of permanent inability to work, or permanent and total disability, dates back to the earliest pension plans of the sixteenth century a serious demand for the estimation of, for example, the probability of a worker becoming permanently invalid at integer age $x$, only arose when the financial liabilities of the German railway pension plans were evaluated in the middle of the nineteenth century (Braun, 1925). No use was then made of the mathematics already in existence for a similar problem.

The earlier German railway pension fund actuaries like Heym and Wiegand $(1859,1865)$, who referred to Heym as the 'creator of invalidity insurance science', were faced with a body of employees who were depleted by deaths and permanent invalids. If $q_{x}$ is written for the probability of death among those presently aged $x$ in this group of active employees, and $w_{x}$ for the corresponding probability of invalidity these authors wrote

$$
p_{x}^{a a}=\left(1-q_{x}\right)\left(1-w_{x}\right)
$$

for the transition probability of an active employee aged $x$ surviving as an active employee through age $x+1$. The two probabilities on the right-hand side of (13) were to be estimated from relative frequencies using observations of deaths and invalidities in the employee body.

We may now extend our use of Hamza's notation to include three further transition probabilities: $q_{x}^{a a}$, the probability of an active individual aged $x$ dying in an active state before age $x+1 ; p_{x}^{a \mathfrak{l}}$, the probability of an active individual aged $x$ becoming invalid and surviving invalid to age $x+1$; and $q_{x}^{a i}$, the probability of an active individual aged $x$ becoming invalid and dying in that state prior to age $x+1$. It follows that

$$
p_{x}^{a a}+p_{x}^{a i}+q_{x}^{a a}+q_{x}^{a i}=1, \quad p_{x}^{a a}+q_{x}^{a a}+w_{x}=1, \quad p_{x}^{a i}+q_{x}^{a i}=w_{x} .
$$

Wittstein (1862) pointed out that the two events whose probabilities are multiplied on the right-hand side of (13) are not independent. Instead he used an estimation formula derived for $q_{x}=1-p_{x}$, the probability of dying at age $x$, for a group of lives subject to accretion and depletion between ages $x$ and $x+1$.

Wittstein (1862) supposes that $A$ lives start the year under observation at age $x, B$ lives enter during the year who were aged $x$ at the beginning of the year, and $C$ similar lives escape from observation. Among the changing group of lives there are $d$ deaths during the course of the year. We are required to estimate $q_{x}$, the probability of death at age $x$.

It is assumed by Wittstein that the two components of the difference $B-C$ are each distributed uniformly over the year so that $(B-C) d t$ can be assumed to occur at epoch $t(0<t<1)$ after the beginning of the year. If, then, $q(x+t, x+1)$ is written for the probability that an individual aged exactly $x+t$ will die in the interval of time $1-t$ before age $x+1$ the expected 
number of lives under observation at age $x+1$ is

$$
A\left(1-q_{x}\right)+(B-C) \int_{0}^{1}\{1-q(x+t, x+1)\} d t=A+B-C-d .
$$

The naive procedure adopted by Wittstein and by actuaries generally until the 1960's was to estimate $q_{x}$ by the quantity derived for $q_{x}$ from this or a similar equation. But before this could be done $q(x+t, x+1)(0<t<1)$ had to be written in terms of $q_{x}$. Wittstein himself chose two alternatives:

$$
\begin{gathered}
q(x+t, x+1)=\frac{q_{x}(1-t)}{1-t q_{x}}, \\
q(x+t, x+1)=1-\left(1-q_{x}\right)^{1-t} .
\end{gathered}
$$

Both these forms assume the values of $q_{x}$ and zero, respectively, when $t=0$ and 1 ; the first is based on the assumption of a uniform distribution of expected deaths over the year of age, and the second assumes a constant force of mortality between ages $x$ and $x+1$. If either expression is inserted into (14) and the logarithmic series developed there results

$$
q_{x} \bumpeq \frac{d}{A+\frac{1}{2}(B-C)}
$$

to a first approximation. Wittstein pointed out that the first neglected term is

$$
-\frac{1}{8}(B-C) \frac{d^{2}}{\left\{A+\frac{1}{2}(B-C)\right\}^{3}},
$$

when (15) is used and is half this for (16). In actuarial practice (18) would be very small.

The approximate relation (14) can be expressed exactly by noting that if $N$ lives are observed for part of their year of life between ages $x$ and $x+1$ such that the putative period of observation for life $j$ is from age $x+a_{j}$ to age $x+b_{j}\left(0 \leqslant a_{j} \leqslant b_{j} \leqslant 1 ; j=1, \ldots, N\right)$, then the expected number of deaths is

$$
\sum_{j=1}^{N} q\left(x+a_{j}, x+b_{j}\right)
$$

and can be equated to $d$, the number of deaths observed. Wittstein's assumption (15) is equivalent to

$$
q(x+a, x+b)=\frac{(b-a) q_{x}}{1-(1-b) q_{x}} \bumpeq(b-a) q_{x}
$$

when the square and higher powers of $q_{x}$ are ignored. A commonly used estimate of $q_{x}$ is obtained by inserting this approximation into (19) to produce (Seal, 1962)

$$
q_{x} \bumpeq d / \sum_{j=1}^{N}\left(b_{j}-a_{j}\right)
$$

The denominator of this expression is called the 'exact duration ' form of the 'exposed to risk' and takes no account of the dates of death of those who die. When all values of $b_{j}-a_{j}$ that are not unity are set equal to one-half, (20) reduces to (17) and the denominator of (20) is called the 'mean duration' form of the 'exposed to risk'. Even in the latter case British and American actuaries have not used the denominator of (17) explicitly but it was named 'the actuarial method' by Berkson \& Gage (1950) and this name unfortunately became incorporated into the statistical literature (Bartholomew, 1973). The concept of exposure to risk as the length of time a person is 'exposed' dates back at least to Woolhouse (1867) but not, I think, to Lambert (1772, pp. $578 \mathrm{ff}$.) even though that author deducted half the smallpox deaths from the original 
group $A$ in arriving at a probability of death from causes other than smallpox. Neverthelessit was Wittstein who gave the 'exposure' idea a probabilistic justification.

Wittstein (1862) argued that if $B$ were zero and the lives escaping from observation consisted of individuals becoming disabled for work one could write $C=A w_{x}$ in (14) and by using (15) obtain the approximate result

$$
p_{x}^{a a}=\left(1-q_{x}\right)\left\{1-w_{x}\left(1+\frac{1}{8} q_{x}\right)\right\}
$$

A similar result held for women subject both to mortality and earlier marriage, reasons for payment of contingent benefits in the early widows benefit plans.

Zeuner (1869) reported Wittstein's article in his book, the second with 'mathematical statistics' in its title, and proposed to evaluate the difference between entrants and exits at epoch $t$ after age $x$ as proportional to $l_{x+t}$, since both types of individual were in some sense 'survivors'. Once again the formula (17) results when squares and higher powers of $q_{x}$ are ignored but it is interesting to note that before expansion (21) becomes

$$
p_{x}^{a a}=\left(1-q_{x}\right)\left\{1-2 w_{x} /\left(2-q_{x}\right)\right\}
$$

Both Wittstein and Zeuner were aware that (21) and (22) assumed that the mortality of active and invalid lives was the same. Kanner (1871) showed how (14) would need modification to recognize the different mortalities of these classes of individuals and, later, Küttner (1880) demonstrated that (22) would need correcting only by replacing $q_{x}$ by $q_{x}^{\prime}$ given by (23) below.

Karup in 1875 began a series of articles to describe the estimation of 'independent' or 'pure' probabilities of mortality and disablement from (11), at this point ascribed to Kanner, if not to Karup himself. If we write

(11) gives

$$
1-q_{x}^{\prime}=\exp \left(-\int_{x}^{x+1} \mu_{x}^{a a} d w\right), \quad 1-w_{x}^{\prime}=\exp \left(-\int_{x}^{x+1} v_{w} d w\right)
$$

$$
p_{x}^{a a}=\left(1-q_{x}^{\prime}\right)\left(1-w_{x}^{\prime}\right)
$$

which, not surprisingly, results also from (6) when the mortality of active lives is the same as that of invalids, namely $\mu_{x}^{a a}=\mu_{x}^{i}$. Karup's major and most pleasing contribution (Karup, 1893) was to estimate $q_{x}^{\prime}$ by

$$
q_{x}^{\prime}=\frac{d}{A-\frac{1}{2} j}
$$

where $j$ is the number of cases of invalidity between ages $x$ and $x+1$, and $w_{x}^{\prime}$ by

$$
w_{x}^{\prime}=\frac{j}{A-\frac{1}{2} d} .
$$

Here $B$ and $C$ of Wittstein's case are supposed to be zero. They can be introduced in the denominators without difficulty. Incidentally, in this paper Karup expressed the forces of exit, for the first time, by single greek letters.

The ensuing furore among German actuaries was dramatic. It has been described at length by Spangenberg (1911) and summarizod by Pasquier $(1913,1915)$ from that account. We limit ourselves to saying that some authors found it difficult to accept the idea of invalidity freed of mortality, one even writing that he would use (25) but not (26); another thought that eliminating one cause of exit would change the rest, a matter already raised in the smallpox controversy; and another argued that the force of a given type of exit would be different when acting alone or in the presence of other forces. The protagonists appeared to feel very deeply about their views and one even called Karup a 'conceited humbug'.

It was well into this century before the 'independent', 'pure', 'partial', 'simple', 'absolute', 
or 'net' probabilities, as they have variously been called, were accepted as error-free concepts. As late as 1925 Berger in his mathematically oriented textbook found it necessary to prove that the use of such probabilities was equivalent to replacing all the actual decrements from other causes by a person of the same age and status as the decrement, a proposition advanced by Karup himself and widely disputed. Richard writing in 1946 said that until very recently the majority of actuaries thought that 'independent' probabilities were only of theoretical interest. One must admit that this is true so long as, for example, a probability of death 'independent' of disability has no intrinsic meaning. This was not the case in the original smallpox problem nor in 'causes of death' problems generally.

Nevertheless actuarial textbooks of many conntries such as Blaschke (1906), Amtmann \& Pfaffenberger (1907), Loewy (1924), Berger (1925), Hunter \& Phillips (1932), Spurgeon (1932), Galbrun (1933), Steffensen (1934), Richard (1946), Böhm (1953), Saxer (1955), Hooker \& Longley-Cook (1957), Dussart (1958), Zwinggi (1958), Risser (1965), Simonsen (1966), Jordan (1967), Coppini (1968) and Wolff (1970) have included substantial sections on multiple decrement mathematics. And there were over 50 articles published on multiple decrement, principally invalidity, mathematics in actuarial journals during the first half of this contury. The only real advances derive from the introduction' of 'revalidity' by Risser (1912) and his reference to a Volterra integral equation for the number of invalids, and from Böhmer's (1912) idea that since

$$
1-q(x, x+1)=\prod_{j=0}^{n-1}\left\{1-q\left(x+t_{j}, x+t_{j+1}\right)\right\} \quad\left(t_{0}=0, t_{n}=1\right),
$$

for estimation purposes the year could be subdivided into $n$ unequal intervals during each of which exactly one decrement occurred. The material included in the actuarial textbooks of those, and even more recent, days consists of the following.

(i) Differential equations are solved for the four fundamental transition probabilities, now extended to $t$ years instead of one:

$$
\begin{gathered}
\frac{d_{t} p_{x}^{a a}}{d t}=-{ }_{t} p_{x}^{a a}\left(\mu_{x+t}^{a a}+\nu_{x+t}\right), \quad \frac{d_{t} p_{x}^{a i}}{d t}={ }_{t} p_{x}^{a a} \nu_{x+t}-{ }_{t} p_{x}^{a i} \mu_{x+t}^{i}, \\
\frac{d_{t} q_{x}^{a a}}{d t}={ }_{t} p_{x}^{a a} \mu_{x+t}^{a a}, \quad \frac{d_{t} q_{x}^{a i}}{d t}={ }_{t} p_{x}^{a i} \mu_{x+t}^{i},
\end{gathered}
$$

with obvious boundary conditions at $t=0$. A 'classic' exposition is that of Böhmer (1914), recently reprinted.

(ii) The approximate simple relations for these transition probabilities, such as (21), (22), (24), are given in terms of either 'independent' or 'dependent' basic probabilities.

(iii) Estimation formulae are given for the basic probabilities, such as (17), (25), (26).

Some of the textbooks (Berger, 1925; Zwinggi, 1958) first developed the mathematics in terms of $n$ causes of decrement and then specialized to the invalidity case which is of special importance to actuaries.

Until the 1930's the mathematics of (i) and (ii) was restricted, with very few exceptions, to publications in German. The British, after Makeham's article assimilating decremental tables with the smallpox literature, limited themselves to the practical problem of estimating the basic decremental probabilities. Sprague (1879), writing just after the most heated publications in the 'independence' dispute and surely with knowledge of it, produced the standard article in English on the subject which showed how to estimate the decremental forces directly by deducting half all decrements in the denominator of expressions such as (25). Alternatively, this could be expressed by saying that the 'exposed to risk' of $(20)$ was to be modified by replacing the $x+b_{j}$ of any decrement by the age at which the decrement occurred. Articles in French, 
even in the Swiss actuarial Mitteilungen, which published the greatest number of contributions to this subject in the twentieth century, are conspicuously absent.

Pesquier's (1913) consideration of a force of 'revalidity' in the differential equations for $l_{x}^{a a}$ and $l_{x}^{i t}$ was shown by him to lead to a second order differential equation of Riccati type which he solved for the particular case of constant decremental forces. It is well known that the Riccati equation is a special case of the Volterra integral equation of the second kind. The latter arises quite naturally as follows.

Hamza (1900) had supposed that $p_{x}^{a a}=l_{x+1}^{a a} / l_{x}^{a a}$ had been obtained for all integer $x$ from observations, e.g. by (21); this, in turn, led to $l_{x}^{a a}$ and then $l_{x}^{i i}=l_{x}-l_{x}^{a a}$ or, independently,

$$
l_{x}^{i i}=\int_{0}^{x} l_{s}^{a a} v_{z} \exp \left(-\int_{s}^{x} \mu_{t}^{i} d t\right) d z
$$

since invalid lives aged $x$ are composed of the survivors of active lives who became invalid at age $z<x$. With $l_{x}^{a a}, v_{x}$ and $\mu_{x}^{i}$ all obtained from observation $l_{x}^{i i}$ could be calculated from (27) by the Euler-Maclaurin expansion, for example. But (27) can be rewritten as

$$
l_{x}=l_{x}^{a a}+\int_{0}^{x} l_{s}^{a a} v_{s} \exp \left(-\int_{s}^{x} \mu_{t}^{i} d t\right) d z
$$

and this is formally a Volterra integral equation of the second kind for $l_{x}^{a a}$ (Risser, 1912). In this equation the mortality of invalids depends on their attained age only but if it is also a function of the time since invalidity, so that $\mu_{t}^{i}$ is replaced by $\mu^{i}(t, t-z)$, say, in (28), we obtain an equation

where

$$
l_{x}=l_{x}^{a a}+\int_{0}^{x} l_{s}^{a a} v_{s} p^{i}(z, x) d z
$$

$$
p^{i}(z, x)=\exp \left\{-\int_{0}^{x} \mu^{i}(t, t-z) d t\right\},
$$

and must utilize the standard Volterra integral equation solution, namely

where

$$
l_{x}^{a a}=l_{x}+\sum_{j=1}^{\infty} \phi_{j}(x)
$$

$$
\phi_{j}(x)=-\int_{x_{0}}^{x} \phi_{j-1}(t) \nu(t) p^{i}(t, x) d t \quad(j=1,2, \ldots), \quad \phi_{0}(x)=l_{x} .
$$

Schoenbaum (1931) used this procedure to derive the values of $l_{x}^{a a}$ for Czechoslovakian social insurance. It is interesting that, in order to secure seven significant figures in $l_{x}^{a a}$, whereas the summation of the $\phi_{j}$ 's was as short as one term at age 21 , by age 80 sixteen terms were needed. This was a considerable numerical achievement in precomputer days.

There have been about a dozen actuarial articles concerned with Volterra equations like (29) or the Riccati specialization. Both Richard (1946) and Risser (1965) report on these developments.

\section{MAXTMUM LIK RLIHOOD hitTMation}

The first half of the twentieth century thus saw the mathematical theory of invalidity insurance, or of transitions between two states $A$ and $B$, established on a sound base. Galbrun's (1933) monograph on the subject, in which a completely new system of notation was introduced, summarized previous developments in this essentially actuarial field. This work was an introduction to the author's later text (1934) in which attention was paid to the number of returns from state $B$ to state $A$ during a given period of time. The writer has dubbed this 'repeated sickness' and Seal (1970) gives a short history. 
Reference to Galbrun (1933) or the more recent Zwinggi (1958) shows that actuaries thought that the 'expected value' technique of Wittstein, not necessarily given his name, was perfectly adequate for the estimation of $q_{x}, w_{x}, q_{x}^{\prime}, w_{x}^{\prime}$ and other probabilities basic to numerical calculations. It was not until Sverdrup (1965) that maximum likelihood estimation of the transition probabilities was introduced to actuaries but by then statisticians had become interested in transitions between states in continuous time.

In 1950 Neyman devoted 27 pages of his new elementary probability text book to the 'Evaluation of competing risks'. Here we find, for example, the probability that an outgoing 'active' patient will survive a year, say, but in the meantime have suffered a relapse into his original disease, namely become 'invalid', written as

$$
Q_{2}=\frac{\lambda_{8}}{\lambda_{1}+\lambda_{2}-\lambda_{3}}\left(e^{-\lambda_{3}}-e^{-\lambda_{1}-\lambda_{2}}\right),
$$

where we can identify the age-independent forces, or intensities of risk, $\lambda$ by comparison with Pesquier's (1913) formula

$$
p_{x}^{a i}=\frac{\nu}{\mu^{a a}+\nu-\mu^{i}}\left(e^{-\mu^{i}}-e^{-\mu^{a a} \rightarrow}\right)
$$

in which the three decremental forces are assumed to be constant throughout the year of age.

A year later Fix \& Neyman (1951) produced maximum likelihood estimates of the transition probabilities and extended the mathematics to the 'repeated sickness' case. Referring to Sverdrup (1965) we see that the maximum likelihood estimate of a decremental force, assumed constant over the year of age, is that used by Sprague (1879) on intuitive grounds as applicable at exact age $x+\frac{1}{2}$. And by the invariance property of maximum likelihood estimators the estimates of $p_{x}^{a a}, p_{x}^{a i}$, etc., obtained by inserting the maximum likelihood estimates of the decremental forces involved therein, are themselves maximum likelihood estimates. This is all rather disappointing for those who want to demonstrate the superiority of new statistical ideas.

We suppose that the neglect of a substantial actuarial literature by today's much larger population of mathematically trained statisticians is merely irritating. Consider, for example, Chiang's (1961) 'weaker assumption', in developing relations between 'net', i.e. 'independent', and 'crude', i.e. 'dependent', probabilities, namely that, in the notation of the present paper,

$$
\mu_{x+t}^{(i)}=r_{x+t}^{i} \mu_{x+t}
$$

This assumption, now known in the statistical literature (David, 1970) as Chiang's proportionality assumption, was given explicitly in this form by Greville $(1948,1949)$ and was introduced as a reasonable hypothesis even earlier by Meier (1940). But how can actuaries complain when, except for Makeham's lip service in 1874, the original founders of the mathematical model of transitions between states have received no attention whatever in the actuarial literature.

\section{REFHRENOES}

Ammanan, H. \& Prafteandergate, E. (1907). Zur Mathematik der Pensionsversicherung. Jena: Fischer. Bartholomew, D. J. (1973). Stochastic Models for Social Processes. London: Wiley.

Bergrir, A. (1925). Die Prinzipien der Lebensversicherungstechnik. Zweiter Teil. Berlin: Springer.

Berkson, J. \& GaGe, R. P. (1950). Calculation of aurvival rates for cancer. Proc. Staff Meetings Mayo Clinic 25, 270-86.

Berrodur, D. (1766). Esabi d'une nouvelle anelyse de le mortalité causée par la petite vérole, et des evantages de l'inoculation pour la prévenir. Hist. Acad. Roy. Sci., Annéo mocolx, pp. 1-45 of Mémoires.

BLAsOHKE, E. (1906). Vorleoungen über Mathematischen Statistik. Leipzig: Teubner.

BömM, F. (1953). Versicherungsmathematik, I. Berlin: de Gruyter. 
Böнorer, P. E. (1912). Theorie der unabhängigen Wahrscheinlichkeiten. Rapp. 7 me Oong. Intornat. Achu. 2, 327-46.

Böfmer, P. E. (1914). Die Grundlagen der Theorie der Invaliditätsversicherung. Jahrb. Versicherungsmath. 142-67. Roprinted (1968) in $B l$. Doutsch. Qes. Versicherungsmath. 8, 353-79.

BRAUN, H. (1925). Geschichte der Lebensversicherung und der Lebensversicherungstechnit. Nuremberg: Koch.

Chinata, C. L. (1961). On the probability of death from specific causes in the presence of competing risks. Proc. 4th Berkeley Symp. 4, 169-80.

Coppnr, M. A. (1968). Lexioni di Tecnica delle Assicuraxioni Sociali. Rome: Veschi.

Counnot, A. (1843). Exposition de la Theorie des Chances et des Probabilites. Paris: Hachette. Reprinted (n.d.), Rome: Bizzarri.

D'Armanerer (1761). Onziéme Mémoire: Sur l'application du calcul des probabiltiés à l'inoculation de la petite vérole. Oprescules Mathématiques 2, 26-95. (Paris: David.)

David, H. A. (1970). On Chiang's proportionality essumption in the theory of compoting risks. Biometrics 26, 336-9.

Dussant, R. L. G. (1958). Sur le Complexe 'Invalidité-Déce's'. Gembloux: Duculot.

FTx, E. \& Neyman, J. (1951). A gimple stochastic model of recovery, relapse, death and loss of patients. Human Biology 23, 205-41.

GalBRUN, H. (1933). Theorie Mathématique de l'Assurance Invalidité et do l'Assurance Nuptialite, tome 3, fasc. Iv of Traité du Calcul des Probabilités et de ses Applications, Ed. E. Borel. Paris: Gauthier-Villars.

Gambrun, H. (1934). Theorie Mathematique de l'Assurance Maladio, tome 3, faso. VI of Traite du Catoul des Probabuliles et de ses Applications, Ed. E. Borel. Paris: Gauthier-Villars.

Grevtute, T. N. E. (1948, 1949). Mortality tables analyzed by cause of death. Record Am. Inst. Actu. 37, $283-94 ; 38,77-8$.

Hamza, E. (1900). Note sur la théotie mathématique de l'assurance contre le risque d'invalidité d'origine morbide, sénile ou accidentelle. Proc. Troisième Cong. Int. Actu., 154-203. (Paris: Dulac).

Hookmen, P. F. \& LoNGLFY-Cook, L. H. (1957). Life and Other Oontingencies, Vol. 2. Cembridge Univeraity Press.

Howthr, A. \& Phسurps, J. T. (1932). Disability Benefits in Life Inourance Policies. Now York: Aotuarial Bociety.

Jompar, C. W. (1967). Lifo Contingencies. Chicago: Society of Actuaries.

Kanner, M. (1871). Allgemeine Probleme der Wahrscheinlichkeitarechnung und ihre Anwendung auf Fragen der Statistik. J. Coll. Lebens. Versich. Wissers. Berlin. 2, 33-53.

KARN, N. (1931). An inquiry into various death-rates and the comparative influence of cartain diseases on the duration of life. Ann. Eugen. 4, 279-326.

KandP, J. (1893). Die Finanzlage der Gothaiochen Staatsdiener-Witwen-Societat am 31 Dezember 1880. Dresden: Morchel.

Kü TTNER, W. (1880). Zur mathematischen Statistik. Zeits. Math. Phys. 25, 11-24.

Lambihrt, J. H. (1772). Beyträge zum Gebrauche der Mathematik und deren Anwendung, Berlin, Teil 3.

Laplade, P. B. de (1812). Theorie Analytique des Probabilites. Paris: Courcier.

LoEwY, A. (1924). Versichorungsmathematik. Berlin: Springer.

Makmham, W. M. (1867). On the lew of mortality. J. Inst. Actu. 13, 325-58.

MAKEHAM, W. M. (1874). On the application of the theory of the composition of decremental forces. J. Inst. Actu. 18, 317-22.

Mened, J. (1940). Zur Theorie der unabhängigen Wahrscheinlichkeiten. Mitt. Veroin. Schweiz. Veraich.Mathr. 39, 63-74.

Neymar, J. (1950). First Course in Probability and Statistics. New York: Holt.

Pasquire, L. G. DU (1913). Mathematische Theorie der Invaliditïtsveraicherung. Mitt. Verein. Schweiz. Versich.-Mathr. 8, 1-153.

PasquIEr, L. G. DU. (1915). Zur Klarstellung. Mitt. Verein. Schweiz. Veroich.-Mathr. $10,48$.

QUIQUET, A. (1934). Duvillard (1755-1832), notes et référances pouvant servir à se biographie. Bull. Trim. Inst. Actu. Franf. 40, 49-105, 121-74, 210-87.

Riohate, P.J. (1946). Theorie et Pratique des Optrations d'Assurance, tome 2. Paris: Doin.

Risser, R. (1912). Importance, application et calcul des probabilités indépendantes et leura rapports aux eutres mesures statistiques. Rapp. 7 mo Cong. Int. Actr. 2, 369-88.

Risger, R. (1965). Applications de la Statistiqus a la Dimographie et d la Biologio, (revisod by C. E. Traynard), tome 3, fasc. III of Traite du Calcul des Probabilites at de ses Applications, Ed. E. Borel. Paris: Gauthier-Villars.

SAXER, W. (1955). Veroicherungomathematik, I, Berlin: Springer.

Söoznвadm, E. (1931). Note sur la théorie mathématique des assurances contre invalidité. Aktu. V\&dy 2 , 10-36. (Trans. of a paper in Casopis PEst. Mat., 1917.)

Seat, H. L. (1962). Deaths among prospective existings. Proc. Conf. Actu. Publ. Pract. 11, 308-10. 
Skac, H. L. (1970). Probability distributions of aggregate sickness durations. Skand. Aktu.-Tidskr. 53, 193-204.

Smongein, W. (1966). Forsikringsmatomatik. Copenhagen University.

Spanameara, P. (1911). Die Karupsche Theorie der unabhängigen Wahraoheinliahkeiten. Veröff. Detuts. Vereins Versich. Wissens. 20, 91-179.

Spraque, T. B. (1878). On the construction of a combined marriage and mortality table from observations made as to the rates of marriage and mortality among any body of men; etc. J. Inst. Actu. 21, 406-52.

SpURgeon, E. F. (1932). Iife Contingencies. Cambridge University Presa.

Stembrangen, J. F. (1934). Forsikringsmatematik. Copenhagen: Gads Forlag.

SVRrdrup, E. (1965). Fistimates and test procedures in connection with stochastio models for deaths, recoveries and transfers between states of health. Skand. Aktu.-Tidskr. 48, 184-211.

Todhunter, I. (1865). A History of the Mathematical Theory of Probability. Cambridgo: Macmillan. Reprinted 1949, New York: Chelsee.

Trembley, J. (1799). Recherches sur la mortalité de la petite vérole. Ḿ́m. Acad. Roy. Sci. BellesLettres, Berlin, 1796, Classe de Math. 17-38.

WrstergaARd, H. (1932). Contributions to the History of Statistics. London: King. Roprinted 1968, New York: Agathon.

WIEGAND, A. (1859). Mathematische Grundlagen für Eisenbahn-Pensionskassen. Halle: Schmidt.

WrEgaND, A. (1865). Versichorung gegen Erwerbsunfahigkeit. Halle: Werner.

WIтTsthen, T. (1862). Die Mortalität in Gesellechaften mit successiv eintretenden und anssoheidenden Mitgliedern. Arch. Math. u. Physik, 39, 67-92.

WoLFF, K.-H. (1870). Versicherungsmathematik. Vienna: Springer.

Woolmouse, W. S. B. (1867). On the construotion of tebles of mortality. Assur. Mag. de J. Inst. Actu. 13, 75-102.

ZEUNFR, G. (1869). Abhandlungen aus der Mathematischen Statistik. Leipzig: Felix.

ZwTragr, E. (1958). Versicherungomathematik. Besel : Birkhauser.

[Received September 1976. Revised March 1977] 